\title{
Carta de Cuiabá - Mulheres, Médicas de Família e Comunidade, no Brasil - onde estamos e onde podemos chegar?
}

Cuiabá Letter - Women, Family and Community Physicians, in Brazil - where we are and where can we reach?

\section{Carta de Cuiabá - Mujeres Médicas de Familia y Comunidad en Brasil - donde estamos y donde podemos llegar?}

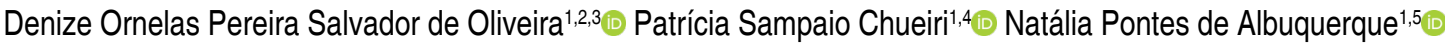 \\ ${ }^{1}$ Coordenadoras GT de Mulheres na Medicina de Família e Comunidade 2017-2019 \\ ${ }^{2}$ Sociedade Brasileira de Medicina de Família e Comunidade \\ ${ }^{3}$ Programa de Residência Médica em Medicina de Família e Comunidade da Secretaria de Saúde de São Bernardo do Campo \\ ${ }^{4}$ Escola de Medicina da Faculdade Israelita de Ciências da Saúde Albert Einstein \\ ${ }^{5}$ Programa de Residência Médica em Medicina de Família e Comunidade do Hospital Metropolitano Odilon Behrens
}

\section{Resumo}

\begin{abstract}
Este artigo é um breve relato histórico sobre a formação do Grupo de trabalho de Mulheres na Medicina de Família e Comunidade da SBMFC (GT-MMFC), que ocorreu em 2016. Em paralelo, descreve-se as principais ações do Wonca Working Party on Women \& Family Medicine e do próprio GT-MMFC até os dias atuais. Os objetivos do artigo são registrar a construção deste grupo de trabalho assim como fomentar e fortalecer o debate de todas as dimensões relacionadas às mulheres e a medicina de família e comunidade e a equidade de gênero.
\end{abstract}

Palavras-chave: Mulher; Equidade de Gênero; Medicina de Família e Comunidade; Atenção Primária à Saúde.

\begin{abstract}
This article is a historical report of the creation of the SBMFC Women's Working Group on Family and Community Medicine (GT-MMFC), which took place in 2016. In parallel, it describes, until the present day, the main actions of the Wonca Working Party Women \& Family Medicine and of the GTMMFC. The objectives of the article are to record the construction of this working group as well as to foster and strengthen the debate on all dimensions related to women and family and community medicine and gender equity.
\end{abstract}

Keywords: Female; Gender Equity; Family Physicians; Primary Health Care.

Como citar: Oliveira DOPS, Chueiri PS, Albuquerque NP. Carta de Cuiabá - Mulheres, Médicas de Família e Comunidade, no Brasil - onde estamos e onde podemos chegar? Rev Bras Med Fam Comunidade. 2020;15(42):1784. https://doi.org/10.5712/rbmfc15(42)1784

\author{
Autor correspondente: \\ Denize Omelas Pereira Salvador de Oliveira. \\ E-mail: denizeofp@gmail.com \\ Fonte de financiamento: \\ declaram não haver. \\ Parecer CEP: \\ não se aplica. \\ Procedência e revisão por pares: \\ revisado por pares. \\ Recebido em: 21/10/2019. \\ Aprovado em: 29/11/2019.
}




\section{Resumen}

Este artículo es un informe histórico de la creación del Grupo de Trabajo de Mujeres SBMFC sobre Medicina Familiar y Comunitaria (GT-MMFC), que tuvo lugar en 2016. Paralelamente, describe las principales acciones del Grupo de Trabajo de Mujeres y Medicina Familiar de Wonca y del GTMMFC, hasta el día de hoy. Los objetivos del artículo son registrar la construcción de este grupo de trabajo, así como fomentar y fortalecer el debate sobre todas las dimensiones relacionadas con la mujer y la medicina familiar y comunitaria y la equidad de género.

Palabras clave: Mujer; Gènero, Sexualidad; Medicina Familiar y Comunitaria; Atención Primaria de Salud

\section{INTRODUÇÃO}

A feminização da medicina é um fenômeno mundial e trouxe à tona a discussão sobre como as questões de gênero atravessam a profissão médica em temas como: a escolha pelas especialidades médicas, diferenciais na remuneração, acesso a posições de liderança e a distribuição de médicas entre os cenários urbanos e rurais. , $2,3,4^{-}$

Segundo a Demografia Médica de 2018 as mulheres representam 45,6\% do total de 414.831 dos profissionais em atividade no Brasil, e já são maioria entre os médicos mais jovens - representam $57,4 \%$ no grupo até 29 anos e $53,7 \%$ na faixa entre 30 e 34 anos. ${ }^{5}$ A diferença no total de profissionais ativos vem caindo a cada ano e aponta para uma crescente feminização da medicina no país.

Há ainda um aumento da presença feminina em quatro das seis áreas básicas da Medicina. Em 2018, na Pediatria, a proporção de mulheres entre os profissionais titulados é de três quartos do total; em Ginecologia e Obstetrícia somam 56,6\%; em Clínica Médica, 52,6\%. Na Medicina de Família e Comunidade as mulheres representam $57,1 \%$ dos profissionais com residência ou título. ${ }^{5}$

Na $21^{\text {a }}$ Conferência Mundial da World Organization of Family Doctors (WONCA), realizada no Rio de Janeiro, em novembro de 2016, mulheres médicas de família, lideranças da Sociedade Brasileira de Medicina de Família e Comunidade (SBMFC) e suas associações estaduais filiadas, se mobilizaram para participar das atividades propostas pelo Wonca Working Party on Women \& Family Medicine (WWPWFM) (Foto 1).

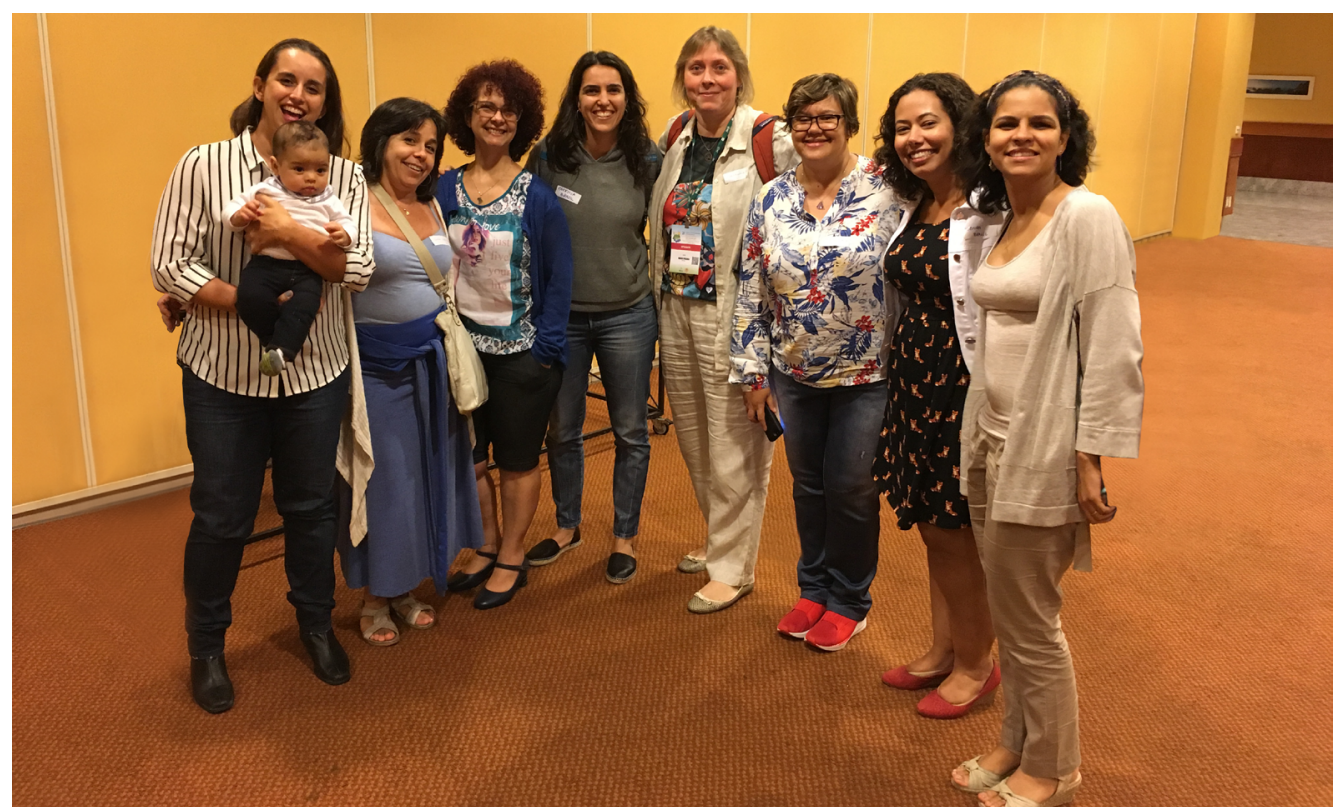

Foto 1: Médicas de Família e Comunidade participantes da 21르 Conferência Mundial da WONCA: Renata Vieira (no colo, Lucas Ornelas), Sara Turcotte, Mônica Lima, Patricia Chueiri, Maria Eugênia Pinto, Zeliete Zambom, Débora Teixeira e Denize Ornelas. 
Entre essas atividades foi discutido como se incorporar as Gender Equity Standards for Wonca Scientific Meetings (GES) aprovadas em 2010 no Conselho Mundial da WONCA em Cancun, México, nos encontros científicos e congressos desenvolvidos pelas sociedades científicas da especialidade. ${ }^{6,7}$

Dessa aproximação e do desejo de difundir entre as médicas de família e comunidades brasileiras as discussões realizadas neste encontro, e de aumentar nosso protagonismo político e representativo, foi fundado em 2016 o Grupo de Trabalho de Mulheres na MFC (GT-MMFC).

O GT-MMFC tem como objetivos discutir a inserção das mulheres na especialidade, planejar ações para romper as limitações decorrentes da desigualdade de gênero no mercado de trabalho e no meio acadêmico, nos âmbitos do ensino e da pesquisa, assim como na própria SBMFC. Desde o seu início, a atuação do GT-MMFC é moldada também junto aos debates com recortes específicos como às questões trazidas pelo GT de Gênero, Sexualidade, Diversidade e Direitos, pela equidade de gênero, contra a cultura do estupro e a homofobia. ${ }^{8,9,10}$

As ações do GT-MMFC também se somaram à iniciativa que as médicas do Grupo de Trabalho de Medicina Rural tiveram desde o Congresso Brasileiro de Medicina de Família, em Natal (2014), e culminaram na publicação do "Manifesto de Natal - Por uma política afirmativa no recrutamento, formação, provimento, retenção e suporte para as médicas de família em contexto rural e remoto". ${ }^{11}$

Nesta sessão especial da edição da RBMFC sobre "Diversidade e direitos humanos na Atenção Primária à Saúde", o GT de Mulheres na MFC da SBMFC traz uma breve história do WWPWFM e disponibiliza documentos pró-equidade de gênero vigentes, já traduzidos, para que médicas de família e comunidade se organizem nas esferas estadual e local.

\section{Sobre o WWWPWFM}

O WWPWFM foi fundado em 2001 na $16^{\text {a }}$ Conferência Mundial WONCA em Durban, assumindo um papel de liderança na defesa das preocupações levantadas por mulheres médicas sobre equidade de gênero na medicina de família e sobre saúde da mulher. Desde então o grupo realiza encontros pré-conferência mundiais, além de oficinas e eventos nas reuniões regionais da WONCA. ${ }^{12}$

Uma das atividades principais do WWPWFM é a proposição e debate de políticas em equidade de gênero como as Hamilton Equity Recommendations - a "Declaração HER”, elaboradas na reunião trienal realizada em Hamilton, Canadá, em 2006, que foram adotados pelo Conselho Mundial da WONCA, em Cingapura, em $2007^{13,14}$ (Quadro 1).

Quadro 1. Recomendações para equidade de gênero - Declaração HER, WONCA, 2007.

1. Inscrever o princípio da equidade de gênero no governo da WONCA alterando os Estatutos e Regulamentos da WONCA, conforme proposto pelo WWPWFM;

2. Implementar a equidade de gênero em todas as atividades da WONCA, em particular programas científicos das suas reuniões trianuais, regionais e rurais;

3. Conferir ao gênero um papel fulcral imprescindível enquanto determinante da saúde;

4. Promover a inclusão equitativa e o progresso de médicas de prática geral/médicas de família na WONCA. 
Além da Declaração HER, o WWPWFM também liderou a elaboração dos "10 passos para a equidade de gênero na Saúde", em Hamilton, 2006, também adotados pelo Conselho Mundial da WONCA, em 2007 (Quadro 2):

Quadro 2. 10 passos para a equidade de gênero na Saúde, WONCA, 2007.

\begin{tabular}{l}
\hline 1. Lutar pela equidade de gênero no acesso aos serviços de saúde; \\
\hline 2. Trabalhar em todas as iniciativas da WONCA para eliminar a violência baseada no gênero; \\
\hline 3. Defender o direito das mulheres à escolha reprodutiva e à maternidade segura; \\
\hline 4. Afirmar o direito das mulheres ao sexo seguro e à escolha sexual; \\
\hline $\begin{array}{l}\text { 5. Defender a participação ativa das mulheres na tomada de decisões e na distribuição equitativa dos recursos (saúde, renda, educação, } \\
\text { habitação, etc.); }\end{array}$ \\
$\begin{array}{l}\text { 6. Integrar a perspectiva de gênero nos currículos e educação médica, treinamento de residência médica, desenvolvimento profissional e } \\
\text { assistência ao paciente durante todo o ciclo de vida; }\end{array}$ \\
$\begin{array}{l}\text { 7. Promover a integração e a compreensão da sexualidade humana saudável no currículo e educação médica, treinamento de residência } \\
\text { médica, desenvolvimento profissional contínuo e assistência ao paciente ao longo da vida; }\end{array}$ \\
\hline 8. Promover e apoiar a investigação sobre o impacto do gênero na saúde; \\
\hline $\begin{array}{l}\text { 9. Incentivar as pessoas e grupos com interesses especiais da WONCA sobre questões como a cessação do tabagismo e abuso de álcool } \\
\text { e drogas, a incorporarem uma perspectiva de gênero; } \\
\text { 10. Reconhecer que a autonomia e empoderamento das mulheres é um fator-chave para tomar medidas concretas sobre o HIV/SIDA e } \\
\text { fazer frente a esta catástrofe em todo o mundo. }\end{array}$ \\
\hline
\end{tabular}

Essas recomendações repercutiram em relevantes mudanças no Estatuto Social e no estabelecimento do Comitê de Equidade Organizacional da WONCA, adotado pelo Conselho Mundial da WONCA, em Cancun, em 2010. Ao assumir este compromisso se afirmou que a atenção às experiências e à condição das mulheres na Medicina de Família e Comunidade é uma importante iniciativa no campo dos direitos humanos. ${ }^{6,7}$ Neste Conselho também foram aprovadas por unanimidade as GES - Gender Equity Standards for Wonca Scientific Meetings, do inglês: "Normas para Equidade de Gênero para os encontros científicos da WONCA" (Quadro 3).

Quadro 3. 10 passos resumidos para Equidade de Gênero para Encontros Científicos da WONCA, 2010.

\begin{tabular}{|c|}
\hline $\begin{array}{l}\text { 2. Conteúdo da programação: incorpora a igualdade de gênero ao longo de sua implementação. Todos os temas das reuniões científicas } \\
\text { incluem uma perspectiva ou análise de gênero. Todos os convites à apresentação de propostas para o plenário, simpósios e workshops } \\
\text { solicitam explicitamente uma consideração de gênero. Sem restrições/viés de gênero na participação. Os temas relacionados à saúde } \\
\text { das mulheres são incentivados, independentemente do tema do encontro. }\end{array}$ \\
\hline $\begin{array}{l}\text { 3. Equilíbrio de gênero: todas as comissões científicas planejam o equilíbrio de gênero para oradores/palestrantes convidados para plenárias, } \\
\text { oficinas e simpósios. }\end{array}$ \\
\hline 4. Palestrantes: mulheres merecedoras são propositalmente e proativamente consideradas como palestrantes. \\
\hline $\begin{array}{l}\text { 5. Bolsas de estudo: a Comissão de Organização ou o comitê de inclusão por bolsas emprega todos os esforços possíveis para aumentar } \\
\text { propositadamente o conjunto de fundos disponíveis e distribuir bolsas equitativamente em relação ao gênero. }\end{array}$ \\
\hline $\begin{array}{l}\text { 6. Desenvolvimento da liderança: as Comissões Científicas empreendem todos os esforços possíveis para promover o desenvolvimento } \\
\text { da liderança em cada conferência. }\end{array}$ \\
\hline $\begin{array}{l}\text { 7. Atividades da família: a Comissão de Organização colabora com os participantes que são mães, pais ou cuidadores de crianças, a fim } \\
\text { de facilitar os arranjos necessários para oferecer cuidado acessível para bebês, crianças pequenas e crianças em idade escolar. }\end{array}$ \\
\hline $\begin{array}{l}\text { 8. Eventos Sociais: a Comissão de Organização garante que todas as atividades sociais oferecidas como parte do programa regular da } \\
\text { conferência respeitam gênero, origem nacional e etnicidade dos participantes e seus convidados, e que humor ou eventos sexistas e/ } \\
\text { ou comentários degradantes não serão tolerados. }\end{array}$ \\
\hline $\begin{array}{l}\text { Patrocínio e marketing corporativo: o Comitê Científico que organiza os encontros da WONCA restringe os patrocinadores externos } \\
\text { ue conduzem políticas ou comercializem produtos que afetam negativamente as mulheres. Todo esforço é feito para restringir imagens } \\
\text { u produtos que objetificam as mulheres ou que fazem alegações enganosas. }\end{array}$ \\
\hline
\end{tabular}


Em 2017 um grupo de mulheres do GT-MMFC aplicou as GES pela primeira vez em um evento científico nacional, no Encontro Mineiro de Medicina de Família e Comunidade (EMMFC), gerando um momento de reflexão e trabalho inicial de adaptação para a realidade brasileira da versão abreviada das Normas para Equidade de Gênero. ${ }^{15,16}$

O checklist adaptado foi apresentado e levado em discussão para a Comissão de Organização do $14^{\circ}$ Congresso Brasileiro de Medicina de Família e Comunidade (CBMFC) realizado em Curitiba no mesmo ano e influenciou vários avanços na condução do congresso, como: a inclusão de um maior contingente de mulheres como palestrantes e de um espaço para lazer de crianças acompanhantes de suas famílias. O estímulo à equidade de gênero nos espaços de congressos também foi incorporado aos Estatutos do CBMFC, aprovado em 2017, determinando que a composição do comitê organizador local e a programação científica devem refletir as políticas de equidade de gênero garantindo a participação de no mínimo $40 \%$ de mulheres e $40 \%$ de homens entre os palestrantes e membros do comitê. ${ }^{17}$

Nos seus dois anos de funcionamento o GT-MMFC vem discutindo pautas relacionadas à atuação e inserção profissional das Médicas de Família e Comunidade, temas como o aborto enquanto problema de saúde pública, ${ }^{18}$ assédio no ambiente acadêmico, violência obstétrica ${ }^{19} \mathrm{e}$ violência de gênero. ${ }^{20}$ Em parceria com o grupo Rural Seeds, foi produzido o vídeo "Participação das Mulheres na MFC" em julho de $2018 .{ }^{21}$

O trabalho do GT-MMFC continuou no $15^{\circ} \mathrm{CBMFC}$ em Cuiabá e onde se realizaram oficinas e atividades propostas como Oficina de Liderança e Mulheres, Diretrizes Sager e Lentes de Gênero, e uma mesa redonda sobre Sexualidade feminina e interseccionalidade, em parceria com o GT de Saúde da População Negra, além da reunião ordinária do grupo ${ }^{9}$ (Foto 2).

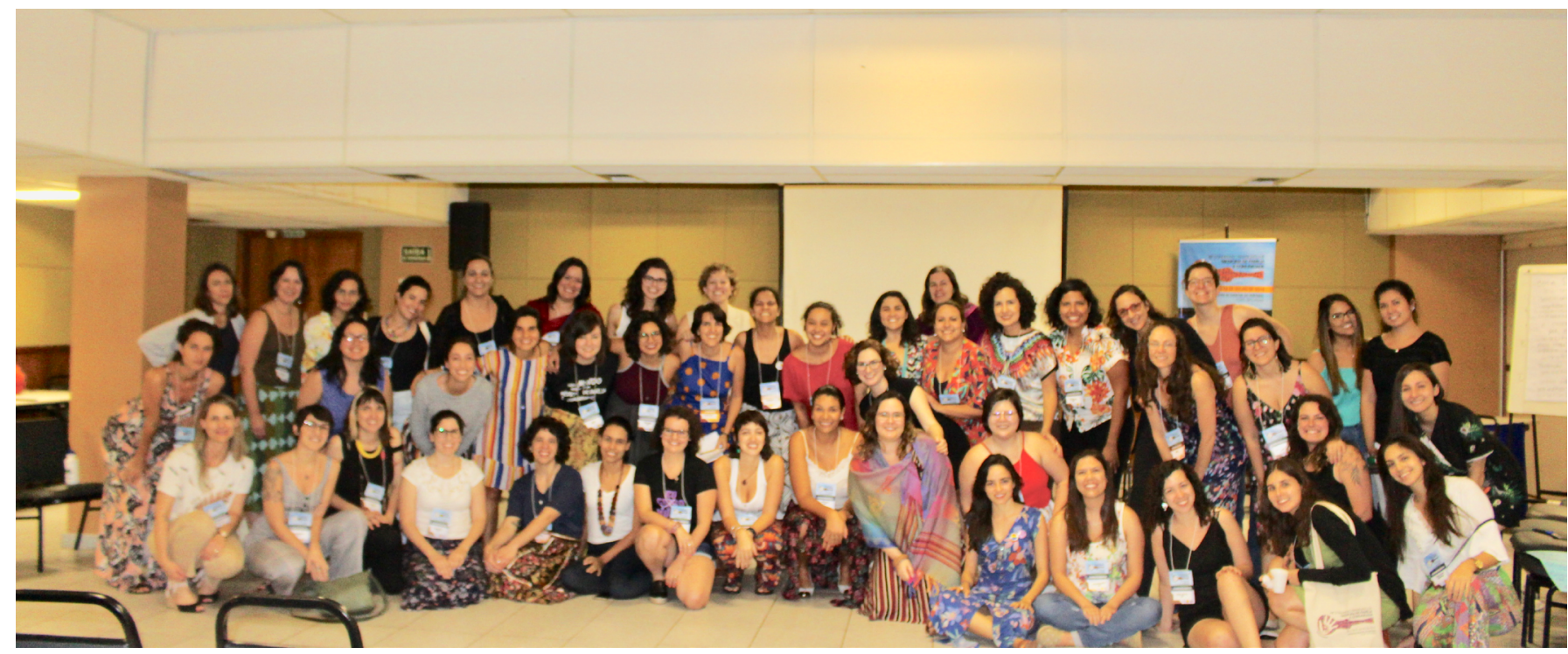

Foto 2: Participantes da Oficina de Liderança e Mulherese da Reunião do GT-MMFC no 15ํㅡㄹㅡ em Cuiabá-MT.

Na Comissão Científica também se buscou ampliar a participação de nós mulheres nas atividades do congresso e neste ano estivemos presentes em 51,5\% das atividades em levantamento preliminar; e foi incluído na revisão da Regulamentação dos Grupos de Trabalho da SBMFC a recomendação da busca 
pela equidade de gênero na composição dos mesmos. ${ }^{22}$

Na oficina de Liderança e Mulheres foi produzido pelas participantes e endossado pelo GT-MMFC, uma carta direcionada à diretoria da SBMFC e seu Conselho Diretor, que reúne os presidentes das entidades estaduais filiadas (Quadro 4). ${ }^{23}$

Quadro 4. Carta de Cuiabá - GT-MMFC da SBMFC, 2019.

Nós, médicas de família e comunidade, residentes e estudantes do Grupo de Trabalho de Mulheres da SBMFC, viemos por meio deste documento propor à SBMFC e suas filiadas, recomendações para fortalecer e apoiar a participação das mulheres nos eventos, nos cargos de gestão e nas pesquisas científicas. Trata-se do resultado da Oficina "Formação de lideranças mulheres para os desafios da MFC", realizada em Cuiabá, em 12 de julho de 2019, durante o 15 Congresso Brasileiro de Medicina de Família e Comunidade - "A saúde em nossas mãos: boas práticas na Medicina de Família e Comunidade".

Considerando que 57,1\% das especialistas em MFC são do gênero feminino, o Estatuto de Congressos Brasileiros de Medicina de Família e Comunidade recomenda:

- $\quad$ A equidade de gênero, crenças e raças como um valor norteador do CBMFC;

- A equidade de gênero na composição do comitê organizador local, e como palestrantes na grade científica (pelo menos $40 \%$ de mulheres e $40 \%$ de homens).

Sugerimos:

- A identificação de gênero no cadastro dos associados e dos membros dos GTs, assim como nos autores da RBMFC;

- Maior participação de mulheres nas mesas relacionadas às políticas de saúde e gestão;

- Maior divulgação dos trabalhos desenvolvidos por médicas de família e comunidade;

- Manutenção de espaços recreativos adequados para crianças de diferentes faixas etárias, nos eventos locais e nacionais, com especial preocupação com a ambiência desses locais, para possibilitar a participação de mães no evento;

- A disponibilização de fraldários nos banheiros masculinos e femininos, bem como espaço reservado para a amamentação com estrutura para ordenha/armazenamento sem representar empecilho para amamentação em todos os espaços;

- Apoiar a divulgação dos canais e espaços de denúncias que o GT de Mulheres desenvolve para a identificação de modalidades de intervenções/atitudes machistas.

É importante registrar que essa edição especial da RBMFC inaugura uma parceria do GT-MMFC e dos editores da revista para estimular os autores a seguirem as Diretrizes Sager no desenvolvimento das suas pesquisas. ${ }^{24,25}$ Esperamos com essa publicação, além de registrar a história e desenvolvimento do GT-MMFC, difundir no nosso país e nas entidades médicas as diretrizes sobre Equidade de Gênero na Saúde, de forma a fomentar os debates que a Diretoria da SBMFC, no sentido da adoção pela entidade de políticas específicas para seus eventos, publicações e espaços de representação.

\section{Declaração de autoria}

Colaboradores: Concepção e/ou delineamento do estudo: DOPSO. Aquisição, análise ou interpretação dos dados: DOPSO, PSC, NPA. Redação preliminar: DOPSO. Revisão crítica da versão preliminar: DOPSO, PSC, NPA. Todos os autores aprovaram a versão final e concordaram em prestar contas sobre todos os aspectos do trabalho, sendo DOPSO, PSC, NPA os acrônimos do nome dos autores.

\section{AGRADECIMENTOS}

Às médicas participantes do GT-MMFC da SBMFC. 


\section{REFERÊNCIAS}

1. Apaydin EA, Chen PGC, Friedberg MW. Differences in physician income by gender in a multiregion survey. $\mathrm{J}$ Gen Intern Med. 2018 Sep;33(9):1574-81.

2. Mainardi GM, Cassenote AJF, Guilloux AGA, Miotto BA, Scheffer MC. What explains wage differences between male and female Brazilian physicians? A cross-sectional nationwide study. BMJ Open. 2019;9(4):e023811. https://doi.org/10.1136/bmjopen-2018-023811

3. Scheffer MC, Cassenote AJF. A feminização da medicina no Brasil. Rev Bioét. 2013 Ago;21(2):268-77. http://dx.doi.org/10.1590/S198380422013000200010

4. Working Party on Rural Practice (WONCA). Política para médicas de família em áreas rurais. Rev Bras Med Fam Comunidade [Internet]. 2013;8(Supl 1):35-48. Disponível em: https://www.rbmfc.org.br/rbmfc/article/view/729

5. Scheffer MC, coord. Departamento de Medicina Preventiva, Faculdade de Medicina da USP. Conselho Regional de Medicina do Estado de São Paulo. Demografia Médica no Brasil 2015. São Paulo: CFM; 2015. 284 p.

6. Lent B, Levitt C, Candib L, Strasser S, Anteyi K, Barnard A, Leopando Z. WONCA's Gender Equity Standards (GES): 10 Years from Conception to Delivery. In: $21^{\text {st }}$ WONCA World Conference of Family Doctors; 2016 Nov 2-6; Rio de Janeiro, Brazil. Rio de Janeiro (RJ): WONCA; 2016; [acesso 2019,outubro,12]. Disponível em: https://gesguidebook.com/wp-content/uploads/2016/10/GES_ Poster_18.10.2016.pdf

7. Wonca Working Party on Women and Family Medicine (WWPWFM). Gender equity standards for Wonca Scientific Meetings. Declaração elaborada pelo WWPWFM em reunião na Universidade de East Anglia; 2009 Jul 2; East Anglia, Norwich, Reino Unido. Norwich (UK): WWPWFM; 2009. p. 1-8; [acesso 2019,outubro,12]. Disponível em: https://www.globalfamilydoctor.com/GetFile.aspx?oid=C34EDB0EB59C-4D4B-A518-09250FA34B7A

8. Levitt C, Lent B, Candib LM, Strasser S, Barnard A, Anteyi K, et al. Nota da Sociedade Brasileira de Medicina de Família e Comunidade pela equidade de gênero e contra a cultura do estupro e a homofobia. Rio de Janeiro (RJ): SBMFC; 2016 Jul 19; [acesso 2019,outubro,12]. Disponível em: http://www.sbmfc.org.br/wp-content/uploads/media/Nota\%20SBMFC\%20pela\%20equidade\%20 de\%20g\%C3\%AAnero\%2019jul\%20AADM(2)(1).pdf

9. Grupo de Trabalho - Mulheres na Medicina de Família e Comunidade (GT-MMFC), Sociedade Brasileira de Medicina de Família e Comunidade (SBMFC). Ata da Reunião Ordinária do Grupos de Trabalho Mulheres na MFC. 15o Congresso Brasileiro de Medicina de Família e Comunidade (CBMFC); 2019 Jul 12; Cuiabá, Mato Grosso, Brasil. Cuiabá (MT): CBMFC; 2019; [acesso 2019,outubro,12]. Disponível em: https://www.sbmfc.org.br/wp-content/uploads/2019/08/Ata-Reuniao-Gt-Mulheres-15CBMFC.pdf

10. Levitt C, Lent B, Candib LM, Strasser S, Bernard A, Anteyi L, et al. WONCA working party for women and family medicine: how to incorporate the gender equity standards (GES) for scientific meetings into WONCA international and regional conferences. In: $21^{\text {st }}$ WONCA World Conference of Family Doctors; 2016 Nov 2-6; Rio de Janeiro, Brazil. Rio de Janeiro (RJ): WONCA; 2016.

11. Lima M, Cuadrado M, Horta TCG, Almeida M, Pinheiro A, Maia P, et al. Manifesto de Natal - Por uma política afirmativa no recrutamento, formação, provimento, retenção e suporte para as médicas de família em contexto rural e remoto. Rev Bras Med Fam Comunidade [Internet]. 2016 Set 19; [citado 2019 outubro 12]; 11(38):1-5. Disponível em: https://rbmfc.org.br/rbmfc/article/view/1247

12. Wonca Working Party on Women and Family Medicine (WWPWFM). Women and Family Medicine. 2019 Jul. Disponível em: http://www. globalfamilydoctor.com/groups/WorkingParties/WomenandFamilyMedicine.aspx

13. Wonca Working Party on Women and Family Medicine (WWPWFM). The Hamilton Equity Recommendations - The HER Statement. Meeting at McMaster University with WONCA; 2006 Aug 24; Hamilton, Canada. Hamilton (Canada): WONCA; 2006. Disponível em: http://www.globalfamilydoctor.com/site/DefaultSite/filesystem/documents/Groups/wwpwfm/THE\%20HER\%20STATEMENT\%202006\%20 with\%20signatures.pdf

14. Lent B. Gender equity - Canadian contribution to international initiative. Can Fam Physician [Internet]. 2007 Abr; ; [citado 2019 outubro 12] 53(3):488-90. Disponível em: https://www.researchgate.net/publication/5969617_Gender_equity_-_Canadian_contribution_to_ international_initiative

15. Albuquerque N, Mendonça M, Fernandes R, Fonseca R, Nascimento A. Método de avaliação de equidade de gênero em congressos: adaptação de ferramenta. Comunicação Oral Coordenada apresentada no 14º Congresso Brasileiro de Medicina de Família e Comunidade; 2017 Nov 2-5; Curitiba, Paraná, Brazil. Curitiba (PR): CBMFC; 2017.

16. Albuquerque N, Mendonça M, Nascimento A, Fernandes R, Coelho B. Equidade de Gênero: aplicação e validação de questionário de avaliação em congressos. Pôster Digital apresentado no 14 Congresso Brasileiro de Medicina de Família e Comunidade; 2017 Nov 2-5; Curitiba, Paraná, Brazil. Curitiba (PR): CBMFC; 2017. 
17. Giugliani C, Ruschel AE, Silva MCB, Maia MN, Oliveira DOPS. O direito ao aborto no Brasil e a implicação da Atenção Primária à Saúde. Rev Bras Med Fam Comunidade [Internet]. 2019 Fev 23; [citado 2019 outubro 12] 14(41):1791. Disponível em: https://www.rbmfc.org. br/rbmfc/article/view/1791

18. Sociedade Brasileira de Medicina de Família e Comunidade (SBMFC). Regimento dos Congressos Brasileiros de Medicina de Família e Comunidade. Curitiba (PR): Conselho de Estaduais da SBMFC; 2017 Out 31; [acesso 2019, outubro,12]. Disponível em: https://www. sbmfc.org.br/wp-content/uploads/2019/10/Regimento-CBMFC.pdf

19. Sociedade Brasileira de Medicina de Família e Comunidade (SBMFC), Grupo de Trabalho - Mulheres na Medicina de Família e Comunidade (GT-MMFC). Nota da Sociedade Brasileira de Medicina de Família e Comunidade a respeito do despacho do Ministério da Saúde que orienta a abolição do termo violência obstétrica em documento oficiais do órgão [carta]. Rio de Janeiro (RJ): SBMFC; 2019 Mai 07; [acesso 2019,outubro,12]. Disponível em: https://www.sbmfc.org.br/noticias/nota-da-sociedade-brasileira-de-medicinade-familia-e-comunidade-violencia-obstetrica/

20. Sociedade Brasileira de Medicina de Família e Comunidade (SBMFC), Grupo de Trabalho - Mulheres na Medicina de Família e Comunidade (GT-MMFC). Série: Dia Internacional das Mulheres - violência contra a mulher. Rio de Janeiro (RJ): SBMFC; 2019 Mar 8-15; [acesso 2019,outubro,12]. Disponível em: https://www.sbmfc.org.br/gt-de-mulheres-na-medicina-de-familia-e-comunidade/

21. Sociedade Brasileira de Medicina de Família e Comunidade (SBMFC). Participação de Mulheres na Medicina de Família e Comunidade [animação/vídeo]. Rio de Janeiro (RJ): SBMFC Oficial; 2018 Jul 14; [acesso 2019,outubro,12]. Disponível em: https://www.youtube.com/ watch?v=rsLaetvMG0E\&t=2s

22. Sociedade Brasileira de Medicina de Família e Comunidade (SBMFC). Regulamentação dos Grupos de Trabalho da SBMFC [carta]. Rio de Janeiro (RJ): SBMFC; 2019 Jun 13; [acesso 2019,outubro,12]. Disponível em: https://www.sbmfc.org.br/wp-content/uploads/2019/10/ Regulamento-GTs.pdf

23. Grupo de Trabalho - Mulheres na Medicina de Família e Comunidade (GT-MMFC). Carta de Cuiabá [carta]. Cuiabá (MT): GT-MMFC; 2019 Jul 12; [acesso 2019,outubro,12]. Disponível em: https://www.sbmfc.org.br/wp-content/uploads/2019/07/CARTA-DE-CUIABA\%CC\%81.pdf

24. Heidari S, Babor TF, Castro P, Tort S, Curno M. Equidade de sexo e gênero na pesquisa: fundamentação das diretrizes SAGER e uso recomendado. Epidemiol Serv Saúde. 2017 Set;26(3):665-76. http://dx.doi.org/10.5123/s1679-49742017000300025

25. Sarti, T. D., Oliveira, D. O. P. S. de, Chueiri, P. S., \& Fontenelle, L. F. (2019). Diversidade e Direitos Humanos na Atenção Primária à Saúde. Revista Brasileira De Medicina De Família E Comunidade, 14(41), 2259. https://doi.org/10.5712/rbmfc14(41)2259 